\title{
El papel de la tecnología desde la perspectiva de Hanna Arendt
}

The role of the technology from the perspective of Hanna Arendt

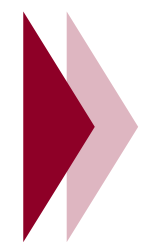

Edisson Díaz Sánchez

Licenciado en Ciencias Sociales de la Universidad Antonio Nariño, Especialista en Gerencia educativa de la Universidad Libre y Magister en Educación de la Universidad Santo Tomás. Actualmente candidato a Doctor en Educación de la Universidad Santo Tomás y docenteinvestigador de la Universidad la Gran Colombia, Bogotá, D.C. (Colombia).

E-mail: edissondiaz@hotmail.com / edisson.diaz@ugc.edu.co

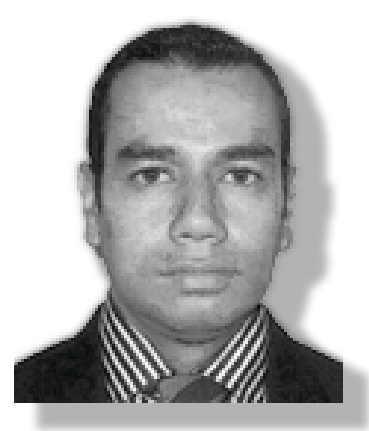




\section{RESUMEN}

El presente artículo de reflexión busca realizar una delimitación del término tecnología, su demarcación conceptual y su relación con el mundo laboral y productivo, a partir de los planteamientos realizados por la teórica política alemana de origen judío Hanna Arendt (2009), en su libro La condición humana, en particular en el capítulo IV: "El trabajo", donde plantea la supremacía del homo laborans sobre el homo faber, desde de una crítica al utilitarismo antropocéntrico de Kant y Protágoras que se refleja en las acciones y actuaciones del homo faber del mundo contemporáneo, para concluir en la importancia de la relación interdisciplinaria entre ciencia-técnica-tecnología dentro de los procesos de desarrollo de las sociedades actuales y la utilización de la tecnología como un medio, más no como un fin de poder, sobrevivencia y calidad de vida.

Palabras claves: Tecnología, Hannah Arendt, trabajo, homo laborans y homo faber.

\section{ABSTRACT}

This article of reflection are looking for a delimitation of the term technology, its conceptual demarcation and its relationship with the world employment and production, from the statements madebytheoretical policyGerman ofJewish origin Hannah Arendt in her book the Human Condition, in particular in chapter IV: "The work", which raises the supremacy of the homo laborans about homo faber, from a criticism of the anthropocentric utilitarianism of Kant and Protagoras is reflected in actions and proceedings of the homo faber of the contemporary world, to conclude on the importance of the interdisciplinary relationship between science - technical - technology within the processes of development of contemporary societies and the use of technology as a means, not as an end of power survival and quality of life.

Keywords: Technology, Hannah Arendt, work, homo laborans and homo faber.
Antes de ser hombres de ciencia, deberíamos ser hombres.

Albert Einstein.

\section{Introducción}

Cada ser humano, en su trayectoria de vida y de formación cultural, va mentalizando y proyectando un estilo de existencia, que necesariamente aborda la manera como visualiza la ciencia, la investigación y el conocimiento científico; es decir, que cada uno de nosotros posee un imaginario colectivo mediante el cual expone su concepción del mundo científico, sea de una cultura o de otra, de una etnia, condición sociopolítica diferente, etc. Pero en este transcurso vital, entramos en choques cognitivos, que nos llevan a dar cambios en nuestro modo de pensar y repensar el micro y el macrocosmos (reingeniería o desaprensión). Situación similar ha sucedido en la historia de la ciencia, que en ciertos momentos históricos, políticos, económicos y culturales ha desarrollado revoluciones científicas, tal como lo plantea Kuhn (1962).

El paso de la modernidad/colonialidad a la posmodernidad y transmodernidad, conceptos analizados por Ramón Grosfoguel, citando a Enrique Dussel (2007), empieza a plantear una serie de discusiones epistemológicas, disciplinarias, científicas, ontológicas, éticas y estéticas entre los diferentes campos y áreas del conocimiento, haciendo énfasis en que existe una ruptura entre las concepciones y nociones de conocimiento, de vida, de educación, de trabajo, de saberes y de prácticas en estos dos estadios de desarrollo cronológico y evolutivo de la humanidad. Desde esta perspectiva, la ciencia, la tecnología y la técnica, y su relación con el mundo laboral y productivo también empiezan a discutirse, no sólo en el campo epistémológico, sino práctico, ético, ambiental, jurídico, entre otros; quizás porque las líneas de demarcación de los campos de acción no estaban claramente definidas o porque los cambios de sociales, culturales, económicos y políticos han afectado de forma directa estas relaciones y definiciones.

Con base en el anterior contexto planteado, el presente artículo de reflexión busca realizar una delimitación del término tecnología, su 
demarcación conceptual y su relación con el mundo laboral y productivo, a partir de los planteamientos realizados por la teórica política alemana de origen judío Hanna Arendt (2009), en su libro La condición humana, en particular en el capítulo IV: "El trabajo", donde plantea la supremacía del homo laborans sobre el homo faber, desde de una crítica al utilitarismo antropocéntrico de Kant y Protágoras que se refleja en las acciones y actuaciones del homo faber del mundo contemporáneo.

Al ser un artículo no derivado de procesos de investigación, la metodología utilizada es de revisión bibliográfica, donde a partir de una selección de textos relacionados con el tema de indagación, se analizan los preceptos fundantes de este escrito y se asume una postura filosófica, analítica y crítica al concepto de tecnología y su materialización en nuestro contexto postmoderno.

\section{Desarrollo}

Como se esgrimió en los anteriores párrafos, el presente escrito empezará con la delimitación conceptual del término tecnología, para luego pasar a su concepción desde los planteamientos de Arendt y su relación con el mundo del trabajo. Recientemente algunos tratadistas como Kranzberg (1991) afirman que la tecnología, como fenómeno cultural y producto socio-científico, es el conjunto de conocimientos, saberes, habilidades y destrezas que han hecho posible la transformación de la naturaleza por el hombre hacia la creación de un mundo artificial, y que son susceptibles de ser estudiados, comprendidos $\mathrm{y}$ mejorados por las generaciones presentes $\mathrm{y}$ futuras.

A partir de lo anterior, se puede asumir la tecnología como un campo de naturaleza interdisciplinaria, entendida por Zabala como "la interacción entre dos o más disciplinas que pueden ir desde la simple comunicación hasta la integración recíproca de contenidos fundamentales y de la teoría del conocimiento" (1999, p. 27); constituido por el conjunto de conocimientos inherentes a los instrumentos que el hombre ha creado, que son aquellos que sirven para algo, y le dan un sentido de intencionalidad a la tecnología, como producción humana. Por lo tanto, el diseño de éstos es un proceso de reflexión y acción, mediante el cual, el individuo relaciona diversos tipos y niveles de saberes, procedentes de las ciencias naturales o sociales, el arte, las matemáticas, entre otros, en la búsqueda de soluciones posibles a problemas reales que afrontan las sociedades y los seres humanos en contextos específicos.

La tecnología, como campo complejo y sistemático de conocimientos, tiene su base en la actividad empírica, técnica y científica. Es necesario entonces, decir que los avances científicos son posibles gracias a los adelantos tecnológicos, ya que el binomio ciencia-tecnología constituye un poderoso factor de transformación de las fuerzas de la naturaleza y la cultura. Este fenómeno se manifiesta en los cambios ocurridos en las relaciones entre los seres humanos, en las variaciones en las relaciones de mujeres y hombres con la naturaleza, en las transformaciones en los ambientes y los cambios en el mundo del trabajo; cambios que en su conjunto constituyen la llamada revolución científico-tecnológica.

Desde la tecnología se diseñan los instrumentos, lo que implica la reflexión y la potencialidad de la creatividad humana. Por ello, el campo de acción de la tecnología abarca lo general, lo particular y lo específico del saber implícito en los artefactos, sistemas y procesos. Por esta razón, la tecnología subsume a la técnica, cuyo campo de acción es particular; es por eso, que mediante ésta actividad sea posible materializar los instrumentos, dado que está referida a las acciones procedimentales para el uso de herramientas, materiales y equipos; es decir, a la manera o modo particular de hacer las cosas (Ministerio de Educación Nacional, 1996).

Una vez puesta en escena la conceptualización y la delimitación reciente que se tiene sobre la tecnología, se pasará a tratar este término dentro de los planteamientos de Arendt (2009) en su célebre escrito de La condición humana. Este texto fue elaborado en 1958, donde estudia el estado de la humanidad en el mundo contemporáneo y hace referencia tanto a las capacidades humanas esenciales para la vida, como a la capacidad de ser libre. En el capítulo IV: "El trabajo", plantea como 
tesis central la supremacía del homo laborans sobre el homo faber, a partir de una crítica al utilitarismo antropocéntrico de Kant y Protágoras que se refleja en las acciones y actuaciones del homo faber del mundo contemporáneo. Aunque, previamente es importante señalar que Arendt antes de caracterizar y diferenciar el actuar en el mundo del trabajo de estos dos seres, propone el término vita activa, para designar tres actividades humanas fundamentales: la labor, que habilita como todo lo que realiza el cuerpo a partir de sus funciones vitales y que le permiten subsistir, donde se ubica la actividad del pensar; el trabajo, referente a todo lo que se realiza con las manos, que está ligado a la voluntad de trascender; y la acción, que es la condición por la cual la vida política se materializa entre los hombres, donde prevalece el juicio y el discurso. Son fundamentales, porque cada una de ellas corresponde a las condiciones básicas en las que el hombre le es dado vivir en la Tierra y que determinan su grado de dominación o libertad.

Para establecer esta distinción, el texto en un primer momento plantea claramente las diferencias axiomáticas entre el homo faber y el homo laborans. Con base en lo anterior, a continuación, se hará un paralelo entre estos dos seres de la actividad del trabajo, a partir de unos ámbitos de comparación.

En términos de definición y conceptualización, homo faber traduce como "el que fabrica y trabaja sobre", mientras que homo laborans es "el que labora y mezcla con". Desde esta perspectiva, el primero es el inventor de instrumentos y útiles que con la ayuda de las máquinas ha transformado el mundo natural en artificial (creador del artificio humano), pero que con el mismo se ha convertido en un peligro para sí mismo y para el mundo, debido a la destrucción de la naturaleza; mientras que el homo laborans se sustenta en la reificación de la labor, es decir que el laborar es un principio de la vita activa que se reconstruye cíclicamente con el movimiento del cuerpo y que le da significado a la existencia humana.

En la esfera del mundo político, el homo laborans se diferencia del homo faber, en la medida que para el segundo el ámbito de lo político se convierte en lo público y este en las relaciones de producción, por lo cual, las relaciones sociales que hace el homo faber se dan en el intercambio de productos y en el valor comercial que a éstos se les dan en el mercado de cambio, donde produce objetos de permuta y no cosas de uso; mientras que el homo laborans, el cual según Arendt (2009), se puede asemejar al artesano de la edad media, tiene en el aislamiento de la fabricación de su idea, su punto de encuentro consigo mismo y con los demás, por convertirse en fabricante, pero al mismo tiempo en maestro de sus aprendices.

Desde la perspectiva teleológica, también existen diferencias entre los fines y los medios entre el homo faber y el homo laborans. El homo faber al creerse dueño y señor de la naturaleza y sus elementos, sostiene que la fabricación tiene un comienzo y un fin definido predictiblemente, ya que su razón presupone que todo se puede establecer a partir de leyes y formulas objetivas creadas por la humanidad para un mundo objetivo, el cual puede ser diseñado y controlado por él mismo. En cambio, el homo laborans piensa que la labor carece de principio y fin, la racionalidad propia del ser humano es ambigua, puede equivocarse en las leyes conformes dela naturalezay que las fronteras entre medio y fin pueden llegar a ser efímeras, ya que todo aquello es cíclico; es decir, lo que es un fin en este momento, después se convierte en un medio para otro fin y así de manera sucesiva, porque al igual que sostenía Platón (2003), desde su concepción de vida contemplativa, para el homo laborans lo que prevalece en la obra de la labor es la idea no la obra misma, y aunque esta esté terminada y por efectos de trasformación su apariencia se modifique, la idea eterna estará presente en ella y en la mente del hombre.

En ese proceso de medio y fin, los medios se materializan en instrumentos, de los cuales el homo faber se adueña en el proceso de fabricación y pierde la perspectiva si son ellos medios para su fin deontológico o son su propio propósito, como ha llegado a suceder con las máquinas, especialmente a partir de la revolución industrial del periodo histórico de la modernidad. En este devenir de conveniencia y utilidad de los bienes, instrumentos y máquinas creados y fabricados por el homo faber, éste se sitúa dentro el utilitarismo antropocéntrico, sistema filosófico que expresa que "durante el proceso de trabajo, todo se juzga en términos de conveniencia y utilidad para el fin deseado, y para nada más" (Arendt, 2009, p. 172). 
Así surge la crítica que Arendt hace alos postulados de Protágoras y Kant, al considerar que ambos sustentaban su sistema antropológico y ético en un antropocentrismo; es decir, en considerar al hombre como el centro y la medida de todas las cosas (fin en sí mismo) y no a la naturaleza o dios, como lo esboza Platón en Leyes (2003), lo cual conlleva a lo que Arendt denomina "el dilema de la no-significación”, mediante el cual el homo faber se convierte en banáusico o filisteo, ya no es capaz de entender el significado de su trabajo y de los útiles e instrumentos que fabrica fuera del mero pensamiento instrumentalista, utilitarista y de conveniencia, lo cual conlleva a perder el valor intrínseco e independiente de los objetos, situación que si es bien comprendida por el homo laborans.

Este proceso de instrumentalización nos llevó al manejo de la tekné, que es entendida como habilidad mediante la cual se hace algo, se transforma la realidad natural en una artificial, conformando así un modelo de construcción y que se puede aprovechar en la enseñanza del mismo para perpetuar esta habilidad. Según Arendt (2009), "El verdadero trabajo de fabricación se realiza bajo la guía de un modelo, de acuerdo con el cual se construye un objeto" (p. 161). En el sentido que lo habla Heidegger, la pregunta por la técnica no es la pregunta por el hacer, sino por la esencia de la técnica, que se espera nos lleve a la verdad sobre las relaciones y las consecuencias (2007).

Es en este dilema teleológico donde surge el acercamiento al problema de la tecnología. Arendt (2009), expone que la tecnología de la época moderna se puede entender desde que "el reemplazamiento de útiles e instrumentos por maquinaria, han surgido a la luz sólo en su última etapa, con la llegada de la automatización" (p. 167). Para ello, la autora describe tres etapas del moderno desarrollo tecnológico desde el comienzo de la época moderna:

1. La invención de la máquina de vapor en la revolución industrial que tuvo su insumo en la explotación de las minas de carbón,

2. El uso de la electricidad y

3. La automatización, la cual ilumina toda la historia del maquinismo.
En este contexto, surge la crítica de Arendt al uso que el homo faber le da a la tecnología, ya que ha convertido las fuerzas y elementos de la naturaleza en medios para satisfacer las necesidades del artificio humano, donde surge el concepto de automatización, el cual es diferente a automático, es decir "todos los movimientos que se mueven por sí mismo, y por lo tanto, al margen del alcance de la deseada y determinada interferencia" (2009, p. 169). Desde esta perspectiva instrumentalista y automatizada, la tecnología pasa a convertirse en un proceso negativo para la vida, la supervivencia del ser humano y de la naturaleza de la tierra, debido a que esos instrumentos, útiles y máquinas que utiliza el homo faber para su labor y cuyas materias primas son de orden natural, terminan acabando y atropellando el fin deontológico para las cuales fueron creadas, ocupando su tiempo en proyectar, usar y contemplar sus obras.

Algunos filósofos, economistas, ingenieros, ambientalistas entre otros académicos, sostienen que el problema central de la tecnología es el servicio que las máquinas prestan al hombre y no la magnitud de las acciones y sus repercusiones en la vita activa del ser humano, como lo plantea Arendt (2009); es decir, que la discusión sobre la tecnología debería girar hacia un problema teleológico y no deontológico, por lo cual

La cuestión, por consiguiente, no es tanto saber si somos dueños o esclavos de nuestras máquinas, sino si éstas aún sirven al mundo y a sus cosas, o si, por el contrario, dichas máquinas y el movimiento automático de sus procesos han comenzado a dominar e incluso a destruir el mundo y las cosas. (p. 170)

Lo anterior afirmación supone una intervención de los procesos de pensamiento que se desarrollan en el ser humano, es decir, que no puede existir una acción tecnológica sin antes haberse desarrollado una acción mental, por lo cual, para Arendt, existe una diferenciación entre pensamiento y cognición, la primera dando origen a las obras de arte y la segunda a las ciencias, cuya afinidad está relacionada respectivamente con el homo laborans y el homo faber; así para el pensamiento no hay un fin u objetivo último ni utilitario como sucede con la labor que realiza el homo laborans en la fabricación de sus herramientas que para él se 
asemejan a una obra de arte; contrario a lo que sucede con la cognición bajo la cual el proceso tiene un fin definido y una vez alcanzado finaliza muy parecido a lo que realiza el homo faber en su labor de medios y fines para el llamado artificio humano. Pero estas dos acciones no se deben confundir con el razonamiento lógico que "se manifiesta en operaciones tales como deducciones de principios axiomáticos o evidentes, inclusión de casos particulares en reglas generales, o las técnicas de alargar consistentes series de conclusiones" (2009, p. 188). El poder de desarrollo de este razonamiento lógico, que es individual, procesual y progresivo en cada individuo conlleva a lo que se denomina "inteligencia".

En términos generales no se trata de percibir si la ciencia, la técnica y la tecnología son buenas, malas o neutras; sino del uso y manejo que el ser humano, a través de su razonamiento lógico, hace de ellas para la construcción permanente de la vita activa. Las consecuencias que actualmente tenemos del avance tecnológico se han visto reflejadas en la velocidad informática, el aprovechamiento del tiempo como recurso no renovable y de riqueza, el crecimiento industrial, la disminución de costos, y grandes ventajas que todas las sociedades han disfrutado tras el paso de las etapas de mejoramiento tecnológico. Pero, ¿será que esas ventajas de la tecnología eran lo que realmente se encontraba oculto, o en realidad es la esclavitud en la que nos hemos prostrado y de la cual no se puede y quiere escapar?; es decir, ¿pensamos que somos homo laborans pero en el fondo seguimos siendo homo faber?

Para algunos críticos de este proceso, la tecnología nos tiene atrapados en sus útiles, instrumentos y máquinas que nos ha llevado a la automatización no sólo de la cognición sino de la vida práctica y no nos deja reflexionar desde un ámbito del pensamiento, para colocar en primer orden la vida y naturaleza de la tierra como factor indispensable para que existan otros medios del artefacto humano. En esta dilucidación, los medios han enaltecido el fin, hasta perder el norte de los avances y de la vida, no debe ser antinatural el progreso, ya que se percibe como implícito del ser humano, pero, ¿será que el crecimiento y avance tecnológico significa un retraso en otros aspectos? De este modo, el dejar de reflexionar sobre lo que se está realizando es un paso gigante hacia la deshumanización de la sociedad.

\section{Resultados y discusión}

En primer orden es necesario entender una nueva visión de la tecnología, no solo como la mera aplicación de la ciencia en un conjunto de aparatos, herramientas, elementos, máquinas, útiles, sistemas, entre otros, que le facilita la vida al ser humano; sino que la tecnología está en estrecha relación con la ciencia y la técnica, donde sus principios, diseños y aplicaciones se hacen para suplir necesidades humanas reales, concretas y situadas, y de este modo mejorar la calidad de vida de los habitantes del planeta.

Lo segundo, se relaciona con el manejo que el ser humano le da a la tecnología en su vida, ya que ontológicamente esta no es mala, sino que el problema se da en su aplicabilidad desde los fines e intereses del hombre, que desde un uso negativo e individualista, conlleva a problemas como la automatización, el daño ambiental, la destrucción de los recursos terrestres, la crisis existencial del ser humano, los conflictos bélicos y la desesperanza por la vita activa.

Un tercer aspecto está relacionado la interacción de la tecnología con el mundo del trabajo, donde en párrafos anteriores se señalaron las diferencias entre el homo laborans y el homo faber, donde el segundo desarrolla la cognición para el abordaje de las ciencias y se centra en una concepción utilitarista del mundo laboral, lo cual conlleva a la automatización; mientras que el primero centra su actividad en el pensamiento para crear objetos que sean obras de arte, donde lo primordial sea la creatividad y satisfacción, y no el lucro o plusvalía que se pueda obtener de su comercialización. Desde esta perspectiva, la tecnología y su relación con el trabajo, debería ser vista desde la óptica del homo laborans, cuyos aparatos, elementos, máquinas, útiles, sistemas sean producto del pensamiento, satisfagan las necesidades humanas y no las del mercado, y donde el trabajador sienta pasión por su labor y que esta sea una prolongación de su vita activa. 
Por último y no menos importante, en la actual sociedad del conocimiento como lo menciona Maiztegui (2002), es primordial desarrollar en todos los niveles de la educación formal, no formal e informal una educación tecnológica y para el trabajo, especialmente a través del desarrollo de competencias tanto tecnológicas como laborales, que permitan que el ser humano potencie sus capacidades, virtudes, habilidades, valores y saberes que le ayuden a un mejor vivir consigo mismo, con la comunidad y con el planeta.

\section{A manera de conclusión}

La condición humana, obra de Arendt, escrita hace más de medio siglo, criticada por varios académicos y avalada por otros, hoy en día sigue teniendo vigencia, a pesar de las diferencias contextuales. Así, la vita activa, propone el triunfo del homo laborans sobre el homo faber; donde en el primero, la labor está asociada con los procesos biológicos que le son inherentes por naturaleza y que le permiten pensar en su bienestar consigo mismo y con el hábitat, tratando de no romper con los ciclos vitales. Para el homo faber, como constructor del mundo artificial, a partir de los procesos de industrialización, la naturaleza es un medio, por lo cual, le es difícil conservarla y para controlarla debe crear herramientas que le permitan llegar a este fin, donde cae en el utilitarismo antropocéntrico, convirtiéndose en un peligro ecológico, societal, ontológico y antropológico.

El papel del trabajo que antes fue propiciar la mecanización para llegar a una buena técnica, ahora deberá estar guiado por el bienestar de la humanidad, lo cual significa que sin importar los beneficios económicos que se obtengan del proceso, se debe realizar una proyección de las consecuencias del uso de la tecnología hacia el futuro, pues en esta oportunidad lo que nos ocupa no es laborar para comer, sino trabajar para sobrevivir algunos siglos más en un planeta habitable. Así, para Arendt, el uso de la tecnología debe significar una herramienta de carácter emancipatorio, "que permita que las personas crezcan, que puedan desarrollar las diferentes dimensiones que tiene el ser humano, pero sin utilizarla como un instrumento para someter a otros" (Uicich, 2008, p. 54). Las consecuencias de las que se debe realizar el cuestionamiento están enmarcadas en el uso y el desuso: cel uso de maquinaría y herramientas tecnológicas en forma desmedida y el desuso del pensamiento en pro del futuroy no dela inmediatez, o viceversa? El trabajo del ser humano, es entonces la construcción de un mundo en donde se pueda vivir y la prioridad sea la vida simple y sencilla como fue creada.

De esta manera, son muchas las oportunidades, pero también los peligros que nos presenta la tecnología, ya que desde una visión meramente instrumental, el componente moral y discursivo de su uso e implicaciones se pierde, lo cual es algo completamente contraproducente para la subsistencia y el buen vivir del ser humano con la naturaleza; dando paso a un enfoque mercantil y utilitarista de la tecnología, donde el medio se convierte en fin y el sentido de lo humano pasa a un segundo plano. Es aquí, donde la acción de los científicos, de las diferentes áreas del conocimiento, se ha convertido en uno de los focos generadores de poder más grande de la historia humana, pero con la particularidad de que la acción de los científicos, al no surgir e insertarse en la trama de las relaciones humanas y políticas debido a su perspectiva universal, no tiene la plenitud de significado que ilumina la existencia humana.

\section{REFERENCIAS}

Arendt, H. (2009). La condición humana. Buenos Aires: Paidós.

Grosfoguel, R. (2007). Descolonizando los universalismos occidentales: el pluriversalismo transmoderno decolonial desde aimé césaire hasta los zapatistas. En: S. Castro-Gómez, El giro decolonial. Reflexiones para una diversidad epistémica más allá del capitalismo global (págs. 63-78). Bogotá: Siglo del Hombre Editores.

Heidegger, M. (2007). La pregunta por la técnica y otros textos. Barcelona: Ediciones Folio S.A. 
Kranzberg, M. (1991). Science-TechnologySociety: It's as Simple as XYZ! Theory into Practice, 234-241.

Kuhn, S. (1962). La estructura de las revoluciones científicas. Mexico: Fondo de Cultura Económica.

Maiztegui, A. y. (2002). Papel de la tecnología en la educación científica: una dimensión olvidada. Revista Iberoamericana de Educación, 28-53.

Ministerio de Educación Nacional. (1996). Educación en tecnología: una nueva respuesta para una nueva educación. Bogotá, D.C.: Ministerio de Educación Nacional.

Platón. (2003). Leyes. Diálogos. Madrid: Gredos.

Uicich, S. (2008). Trabajo y tecnología en la crítica de Arendt a Marx ila revolución (im)posible? Bahía Blanca: Universidad Nacional del Sur.

Zabala, A. (1999). Enfoque globalizador y pensamiento complejo. Una respuesta para la comprensión e intervención en la realidad. Madrid: Editorial Graó. 